\title{
Federated Identity Management and Interoperability for Heterogeneous Cloud Platform Ecosystems
}

\author{
Nirojan Selvanathan \\ IoT Group \\ Salzburg Research \\ Salzburg Austria \\ nirojan.selvanathan@salzburgresea \\ rch.at
}

\author{
Dileepa Jayakody \\ IoT Group \\ Salzburg Research \\ Salzburg Austria \\ dileepa.jayakody@salzburgresearc \\ h.at
}

\author{
Violeta Damjanovic- \\ Behrendt \\ IoT Group, Salzburg Research \\ Salzburg Austria \\ violeta.damjanovic@salzburgresear \\ ch.at
}

\begin{abstract}
This paper describes an approach to overcome the interoperability challenges related to identity management systems supporting cross-collaboration between heterogeneous manufacturing platforms. Traditional identity management systems have shown many weaknesses when it comes to cloud platforms and their federations, from not being able to support a simplified login process, to information disclosure and complexity of implementation in practice. This paper discusses workflows to practically implement federated identity management across the heterogeneous manufacturing platforms and design interoperability at different levels, e.g. at the platform level and at the platform integration level. Our motivation to find the best federated identity management solution for heterogeneous cloudbased platforms is related to practical requirements coming from the ongoing European project eFactory.
\end{abstract}

\section{CCS CONCEPTS}

- Information systems $\rightarrow$ Information systems applications • Security and privacy $\rightarrow$ Security services $\rightarrow$ Access control

\section{KEYWORDS}

Federated identity management, Identity management systems, Federation, Digital manufacturing platforms, Manufacturing, Interoperation, Heterogeneous systems, Cloud computing

\section{ACM Reference format:}

Nirojan Selvanathan, Dileepa Jayakody and Violeta DamjanovicBehrendt. 2019. Federated Identity Management and Interoperability for Heterogenous Cloud Platform Ecosystems. In Proceedings of the $14^{\text {th }}$ International Conference on Availability, Reliability and Security (ARES'19). Canterbury, United Kingdom, 7 pages. https://doi.org/10.1145/3339252.3341492

Permission to make digital or hard copies of part or all of this work for personal or classroom use is granted without fee provided that copies are not made or distributed for profit or commercial advantage and that copies bear this notice and the full citation on the first page. Copyrights for third-party components of this work must be honored. For all other uses, contact the Owner/Author.

ARES '19, August 26-29, 2019, Canterbury, United Kingdom

(C) 2019 Copyright is held by the owner/author(s)

ACM ISBN 978-1-4503-7164-3/19/08.

https://doi.org/10.1145/3339252.3341492

\section{Introduction}

This paper presents an approach to designing and implementing a federated identity management and interoperability system for an ecosystem of heterogeneous, cloudbased manufacturing platforms, which is currently being developed in a European research project called eFactory [1]. The project creates a collaborative manufacturing platform ecosystem, enabling the transition from conventional mass-production systems, to lot-size-one manufacturing. It offers support for establishing digital twins for production processes and for optimization and forecasting support in industrial production systems. The platform interlinks four base platforms developed since 2016, and funded by the European Commission as part of the "Factories of the Future" (FoF) programme within Horizon 2020.

The eFactory Data Spine will be designed as an integration and interoperation middleware, enabling various toolsets and services from the base platforms to fully connect with each other and offer their capabilities to the user communities of the four base platforms, and ultimately, to a wider audience via eFactory itself. Thus, finding the best solution for the federated identity management is a crucial issue to be analyzed and solved early in the project, to enable the platform federation and interoperation, and hide the complexity of dealing with different solution providers.

Paper organization. Section 1 explains our motivation to research the federated identity management in the context of heterogeneous, cloud-based platform ecosystems. Section 2 explores the literature on both the traditional IDentity Management (IDM) systems and cloud-based IDMs, including the federated cloud identity broker model and its advancements discussed in the BlindIdM and the privacy preserving federated cloud identity broker models. In Section 3, the high level eFactory requirements related to the federated IDM solutions, are presented. Section 4 is about the design of the federated IDM and interoperability, and introduces the eFactory Security (EFS) portal and its major goals. Section 5 presents our prototype implementation, featuring the two authentication workflows between the eFactory platform and base platforms. Here, we also differentiate between two interoperability implementations: at the platform level and at the platform integration level. The platform level interoperability implementation offers federation login and 
Federated Identity Management and Interoperability for Heterogenous Cloud Platform Ecosystems, ARES'19, August 2629, 2019, Canterbury, United Kingdom

Single Sign On (SSO) capability to the base platforms. The platform integration level interoperability is implemented through service accounts and the eFactory Data Spine. Section 6 gives some conclusion remarks and discusses next steps in our research.

\section{Literature Review on Identity Management Systems}

Recent research directions on IDM refer to the following four models that differ in scalability, privacy and user controls [2]:

The isolated identity model is the simplest, traditional IDM that merges the Service Provider (SP) and the IDentity Provider (IDP), and allows for identification and authentication to be carried out at the SP. In this model, in order to access services of another SP, the user needs to register at the other SP's IDM. Managing of the diversity of credentials for accessing various service providers may become difficult for users [3].

The central identity model allows the IDP to take over all identity-related services for the SP, e.g. identification and authentication, the management of the identity lifecycle, etc. [4]. In this model, the users' identity data are stored in a central repository at the IDP and the SPs do not need to maintain identity data in their own repositories [5]. Some examples of the central IDM models are Kerberos [6] and the Central Authentication Service (CAS).

The user-centric identity model stores all identity data directly in the user's domain, i.e. on a secure token, a smart card, etc. In this model, the user's identity data can be transferred by an IDP to an SP only after the user gives the consent, which tremendously increases users' privacy [7]. Some examples of the user-centric model are the Windows CardSpace project and several national eID solutions such as the Austrian citizen card [8], the German eID [9], etc. One of the major concepts of the Windows CardSpace was an InfoCard, a collection of claims about an identity that could be chosen during an authentication request or switched during communication with an SP.

The federated identity model stores the identity data in a distributed manner, across different IDPs and/or SPs. In this model, it does not exist a single entity that is in full control of the identity information [10]. All IDPs and SPs form a federation and share a common trust relationship amongst each other, which is usually established on an organizational level; the enforcement is carried out through the platform, on a technical level. This model supports identification and authentication across different domains, enabling cross-domain Single Sign-On (SSO) [5]. Popular examples of this approach are the Security Assertion Markup Language (SAML), Shibboleth, or WS-Federation [11]. SAML 2.0 is an OASIS standard that provides an XML-based framework for creating and exchanging security information between the users [12]. The Shibboleth project started as an Internet2 Middleware Initiative in 1999, and was focused on the development of interoperable identity and access management between web-based resources. Shibboleth is an implementation of
N. Selvanathan et al.

the SAML protocol that shows excellent scaling, both in performance and manageability, and can be extended to support custom scenarios [13]. Some other examples of the federated identity models are the federations operated by various National Research and Education Networks (NREN), e.g. IDEM (www.idem.garr.it) by the Italian NREN - GARR, AAF (aaf.edu.au) by the Australian NREN - AARNET, and eduIDM (www.eduidm.ma) by the Moroccan NREN- MARWAN [14].

Research on cloud-based IDM differentiates between the following models [2]:

- the identity IN the cloud model is similar to the isolated identity model in which users' identity data are stored in the domain of the cloud SP. The model minimizes the control of users over their data in the cloud (e.g. Google, Salesforce.com), but does not support a simplified login process (i.e. SSO);

- the identity TO the cloud model is similar to the central identity model. In this model, SP and its applications are cloud-based, whereas the IDP is not deployed in the cloud and data are not disclosed to a cloud SP. The IDP transfers identity and authentication data to the cloud SP through standardized interfaces that are based on SAML, OpenID, OAuth.

- the identity FROM the cloud model is also known as "Identity as a Service Model" [15]. In this model, both the cloud application and the IDP are operated in the cloud by cloud SPs. Some examples of this model are Google Accounts Authentication and Authorization, or Facebook Login.

- the cloud identity broker model is an extension of "the identity FROM the cloud model", in which the IDP acts as a cloud identity broker or a hub between one or more SPs and one or more IDPs [16][17][18]. By introducing the broker concept, this model hides the complexity of the individual IDPs from the SP. In addition, the SP needs to implement only one interface (to the identity broker). Here, there is only one strong trust relationship that is required between the SP and the identity broker. Some relevant examples of this model are McAfee Cloud SSO, the SkIDentity, the Cloud ID Broker, etc.

- the federated cloud identity broker model combines the traditional federated identity model with the cloud identity broker model [18]. In this model, the user and SPs can rely on the individual broker of their choice, which eliminates the drawback of being dependent on the same identity broker.

- the BlindIdM model is another extension of "the identity FROM the cloud model" [19][20]. This model enables identity data storage and data processing to be performed by the semi-trusted IDPs in the cloud, or without knowing the contents of these data. Hence, the IDP provides these data in a blind manner [20], by using a proxy re-encryption scheme $[21][22]$. This is an innovative contribution to the identity management solutions. 
Federated Identity Management and Interoperability for Heterogenous Cloud Platform Ecosystems, ARES'19, August 2629, 2019, Canterbury, United Kingdom

- $\quad$ the privacy preserving federated cloud identity broker model improves privacy preservation for users, by combining the advantages of the "federated cloud identity broker model" with the advantages of the BlindIdM model. This model can be used with semi-trusted cloud identity brokers [23]. It requires two re-encryption steps, since identity data flow through at least two cloud identity brokers. In addition, the user has to generate two re-encryption keys (one for the direction Identity Broker $1 \rightarrow$ Identity Broker 2 and another one for the direction Identity Broker $2 \rightarrow \mathrm{SP}$ ), which makes this model complex to implement. The implementation also requires an appropriate governance model to be put in place, to support the use of proxy re-encryption [23].

\section{3 eFactory Federation and Interoperation Requirements}

One of the major requirements of the eFactory platform is to establish a federation and enable interoperation between four manufacturing platforms, namely NIMBLE $^{1}$, COMPOSITION $^{2}$, DIGICOR $^{3}$, and vf-OS ${ }^{4}$. The four platforms offer a good complementarity of business capabilities, with a relatively uniform technology basis. In order to "combine" the capabilities of the base platforms' services with the resources of other cloudbased systems outside the boundaries of the respective platforms, the eFactory platform must overcome the interoperability challenge that is present at the level of currently existing IDM systems. We follow the conceptual platform interoperability framework that promotes transparency along three levels (i) platform interoperability, (ii) application interoperability, and (iii) platform integration interoperability. The platform interoperability is focused on the separation of 'identification' from 'services' to allow shared access across different platforms. It enables user identification in multiple cloud platforms. The next level of interoperability framework is the application interoperability, enabling cross platform application (service) deployment. Finally, the top level is the platform integration interoperability, which enables seamless integration between cross-platform services through integration middleware.

Regarding the implementation, the interoperability between different IDMs is still to be solved, e.g. in many real-world scenarios, the user needs to provide attributes from multiple IDS, whereas (s)he can only use one IDP within a single SP session [14]. The activity diagram showing the platform integration level interoperability in eFactory is illustrated in Figure 1. To access multiple platforms in the ecosystem and to use a single set of credentials among them, several controls would need to be enabled through integration, e.g. user identity and access control

\footnotetext{
${ }^{1}$ https://www.nimble-project.org/

2 https://www.composition-project.eu/

${ }^{3} \mathrm{https}: / /$ www.digicor-project.eu/

${ }^{4}$ https://www.vf-os.eu/
}

N. Selvanathan et al.

management (preferably based on attributes and/or contextual information), policy management, event management, authentication, command execution at the top level to enable password management, policy configuration by the users, etc. Therefore, to enable the federated identity, the eFactory platform requires the eFactory Security (EFS) portal to be designed and implemented to efficiently govern the security management for different platforms. The EFS is a distributed single point of trust that enables a class of Super Administrator whose role is to provide secure authentication of any tenant platform in the ecosystem (e.g. multi identities to be managed across company's accounts) (see Figure 1).

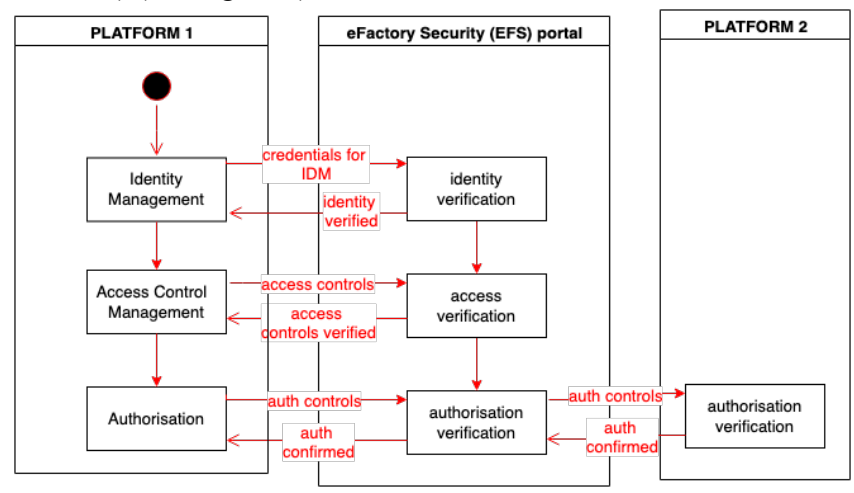

Figure 1: Cross-platform user identity and access management via EFS (platform integration interoperability)

With respect to access control mechanisms for the IoT and the cloud, traditional Role Based Access Control (RBAC) has shown serious weaknesses, e.g. confused deputy attacks through inherited permissions (the user with higher permissions grant access to a specific resource to a user with lower permissions). As an alternative to RBAC, the Attribute Based Access Control (ABAC) model provides fine-grained access mechanisms, in which the authorization decisions are based on attributes that need to be proven by the user (location, roles, etc.) and on other properties (e.g. resource properties) [24].

\section{Federated Identity Management and Interoperation Design}

The eFactory platform and its eFactory Data Spine integration middleware, act as the federation provider that governs identity federation and user provisioning workflows across the eFactory ecosystem. The eFactory Data Spine integration middleware includes the EFS (see Figure 1) as a component that implements a variety of security controls, apart from the federated IDM. Figure 2 illustrates the high level EFS perspective of the integration of base platforms with the eFactory platform. Each base platform contains its own IDP that maintains the platform's users, their roles and access policies. Practically through EFS, the eFactory 
Federated Identity Management and Interoperability for Heterogenous Cloud Platform Ecosystems, ARES'19, August 2629, 2019, Canterbury, United Kingdom

Data Spine federates the existing users of base platforms across the platform ecosystem.

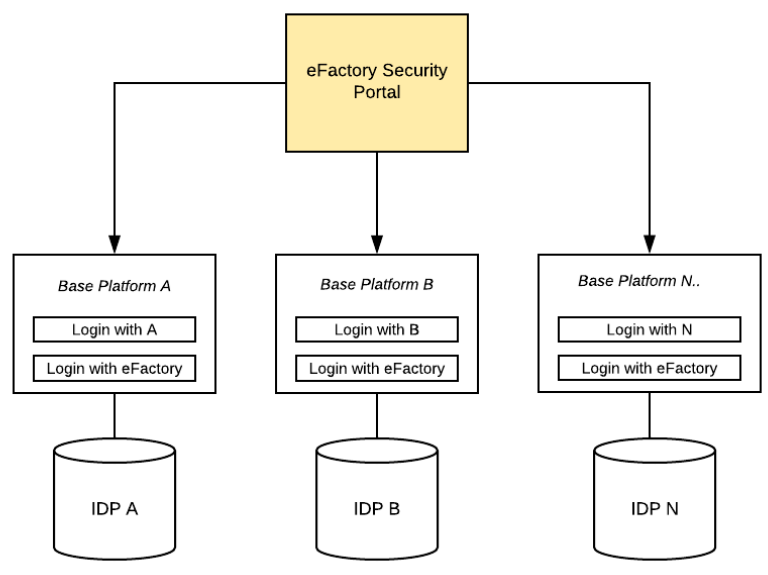

Figure 2: High level design of the eFactory federation

One of the design goals in eFactory is to accommodate not only four base platforms, but also external platforms that will provide further collaborative manufacturing services. With such a design requirement, one-to-one user federation mappings between platforms will result in high number of login options for individual platforms (e.g. login to platform A, B, C, .., N), which will require continuous updates of the authentication and authorization workflows for each platform in the ecosystem that is rather, not feasible.

Therefore, the eFactory Data Spine and EFS take on the role of the central federation provider to manage all federation workflows across platforms participating in the ecosystem. The EFS also acts as the gateway enabling the access to the collaborative manufacturing resources provided by the platforms in the ecosystem. Figure 3 depicts design goals of the EFS, featuring the following capabilities:

Heterogenous Federation Protocol support: Currently all four base platforms have their own IDPs and support OpenID and Oauth 2.0 for authentication and authorization. We envision eFactory to grow as an ecosystem with more manufacturing and supply chain platforms joining the project in future, which requires different identity federation protocols to be supported. Thus, the EFS should support other popular identity federation protocols including SAML, Shibboleth etc.

Policy and Role Governance: The base platforms in eFactory come with the defined user roles and access policies that are managed in their own IDPs. After joining the eFactory ecosystem, base platforms are expected to start collaborating and exchanging data. For example, when the user of one base platform wants to use a tool or a service from another platform, the service access needs to be authorized according to a commonly agreed-upon authorization policy. This requires an intermediary user role and policy governance workflow in EFS, to define access roles and
N. Selvanathan et al.

policies for the users in eFactory, based on their roles and access levels as defined in the base platforms.

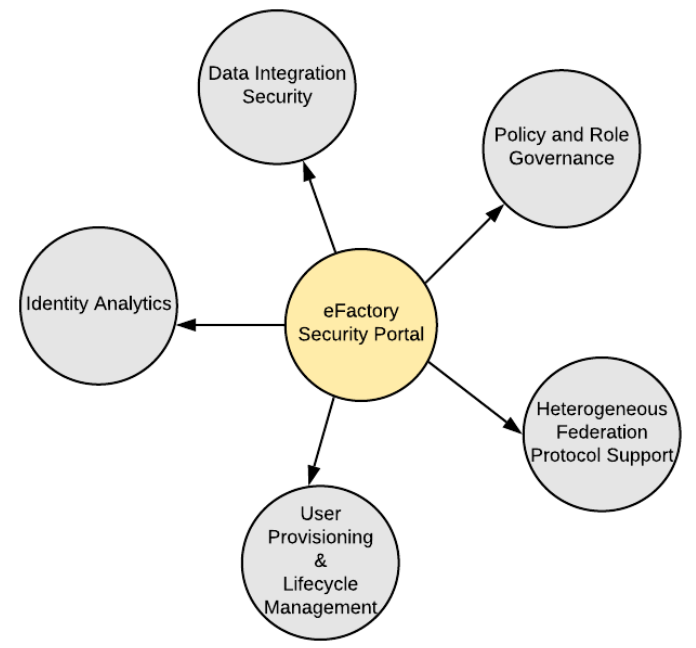

Figure 3: The design goals of EFS

Data Integration Security: As described in Section 3, eFactory aims to provide an interoperability framework to promote collaboration and transparency across different manufacturing platforms. The integration level interoperability requires data security and access control management at service/API level. This requires protocol switching, data enhancement, transformation and loading (ETL) processes and applying security policies at API level, to be controlled by the eFactory Data Spine integration middleware. Thus, data integration level security is another core requirement of the EFS and the eFactory Data Spine.

User Provisioning and Lifecycle Management: Managing and synchronizing the users across different security domains of base platforms, is yet another key requirement of the EFS. When a new user registers in the eFactory portal, (s)he needs to be provisioned in the eFactory domain with relevant authorization policies, in order to access the other platforms' services. With each change to the user's role or the authorization policies in the base platform, the changes should be synchronized through EFS. Therefore, EFS will support user provisioning, synchronization and lifecycle management by implementing the workflows using the System for Cross-domain Identity Management (SCIM).

Identity Analytics: In the enterprise world, statistics and data analytics derived from system data are instrumental to create valuable business insights. Through the eFactory Data Spine, we collect insightful statistics including login attempts from the platforms in or outside of the ecosystem, federated login attempts, distribution of login attempts based on geography, time, behavior of users and users' groups, etc. These data can be further processed through security analytics residing in EFS, created to 
Federated Identity Management and Interoperability for Heterogenous Cloud Platform Ecosystems, ARES'19, August 2629, 2019, Canterbury, United Kingdom

provide relevant security decisions, e.g. potential threats, attack prevention, remediation strategies, etc.

\section{Federated IDM Prototype Implementation}

This section describes the prototype implementation of the eFactory federated IDM at two levels, i.e. platform and platform integration levels.

\subsection{Implementation of the Platform Level Interoperability}

In order to login to one of the four base platforms, the user can select one of the following procedures for the authorization:

1. Login through a base platform (native users), and

2. Login through the eFactory platform (federated users).

The platform level interoperability in eFactory can be achieved by following workflows 1 and 2, shown in Figures 4 and 5, respectively. Note that both workflows require the user to be registered on the eFactory platform.

Figure 4 illustrates the workflow that follows the bottom up approach for federation. Here, the user logins to the base platform using his/her eFactory credentials. The eFactory credentials are issued by the EFS and are not propagated to the base platforms. The initial representation of the user will be created when the user opts to login with eFactory credentials in a base platform. If a user is already present in the base platform then a linked user will be created with the existing roles of the base platform.

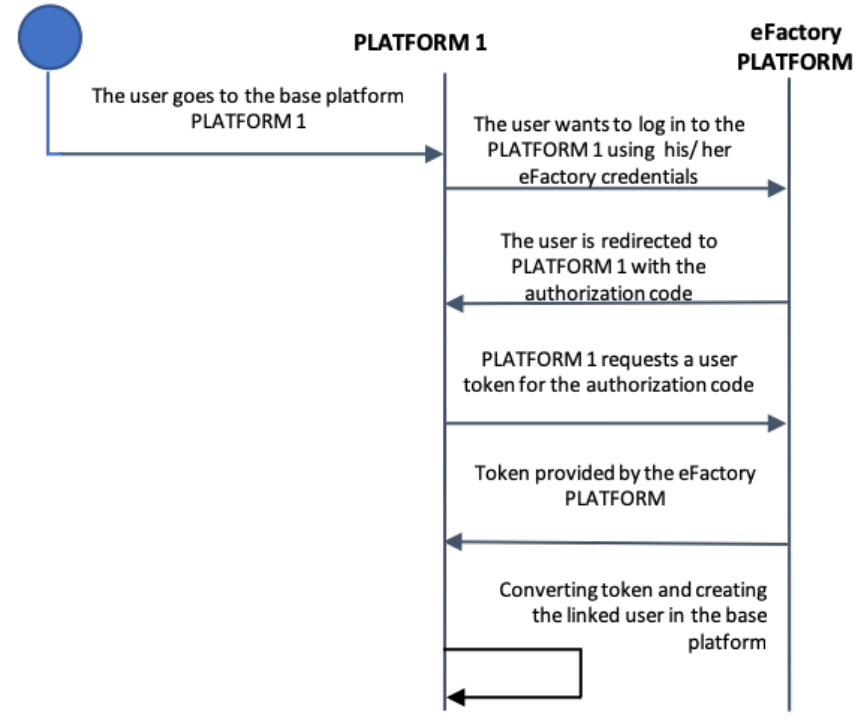

Figure 4: Workflow 1: Login to PLATFORM 1 using eFactory platform credentials
N. Selvanathan et al.

The second workflow enables the user to independently login to the eFactory platform (via EFS) and then visit any base platform in the same browser session, e.g. PLATFORM 1, PLATFORM 2, etc. In this approach, the user logins to the base platforms using his/her previously provided eFactory credentials. By keeping the common browser session, the eFactory user can achieve SSO capability when login to other base platforms.

Figure 5 illustrates the second approach.

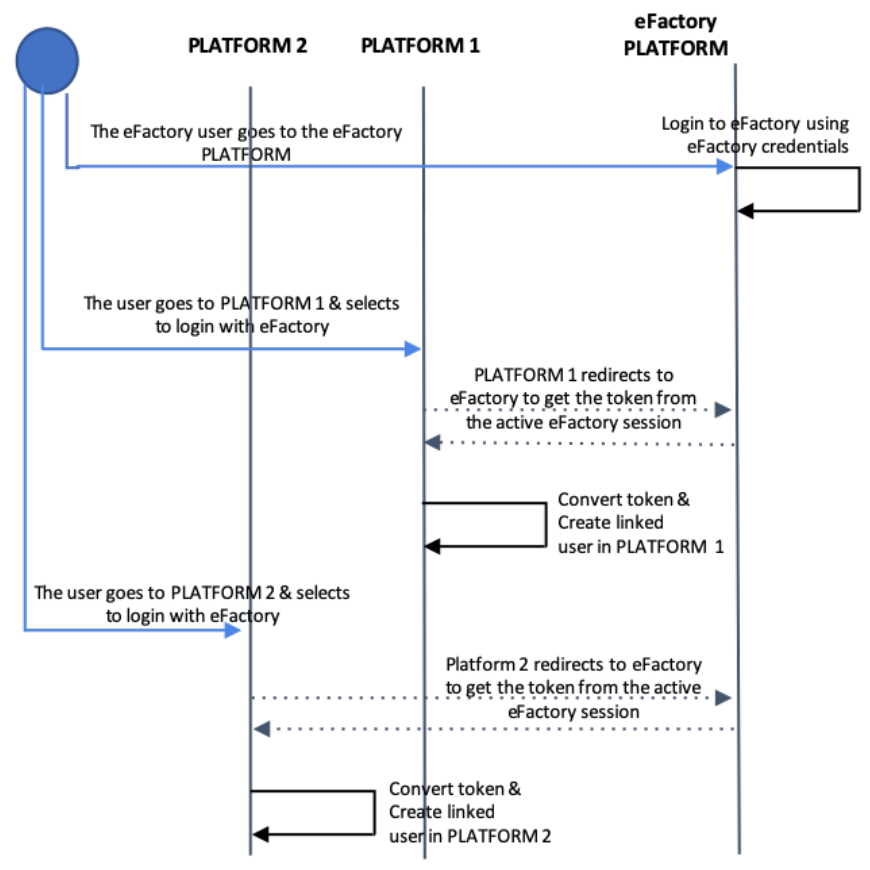

Figure 5: Workflow 2: Login to eFactory using eFactory platform credentials, then login to PALTFORM 1 and PLATFORM 2

The current implementation of the eFactory EFS (with IDS) follows the OpenID based identity federation. Here, the user who tries to login to the base platform using eFactory credentials, is redirected to the eFactory platform (and its EFS) to validate his/her credentials. After verifying the credentials, the user is redirected to the relevant base platform. Furthermore, the base platform identifies the user and provides required roles based on predefined policies.

\subsection{Implementation of the Platform Integration Level Interoperability}

The platform integration level interoperability requires the eFactory Data Spine integration middleware to be put in place, to provide access to the base platforms via service accounts. The policies and access controls between base platforms are mediated and translated by the EFS's policy engine. The OpenID clientbased credentials flow has been implemented to enable access between the platforms. 
Federated Identity Management and Interoperability for Heterogenous Cloud Platform Ecosystems, ARES'19, August 2629, 2019, Canterbury, United Kingdom

In comparison to Figure 4 and Figure 5, which both illustrate the implementation workflow for the platform level interoperability (without a platform middleware), Figure 6 illustrates currently supported integration level interoperability between platforms, participating to the eFactory ecosystem. In Figure 6, the Security Portal (with IDP) is part of the eFactory Data Spine integration middleware between several platforms that need to be integrated. The role of the eFactory Data Spine is to
N. Selvanathan et al.

federate service calls, manage the access to the base platforms services, and provide a global IDM system for eFactory.

The user privilege data, e.g. user roles, are synced from based platform to the EFS, and are used inside the EFS policy engine to grant access to resources in other platforms via the platform integration level interoperation. XACML (eXtensible Access Control Markup Language) policy language has been currently used to formulate the policies.

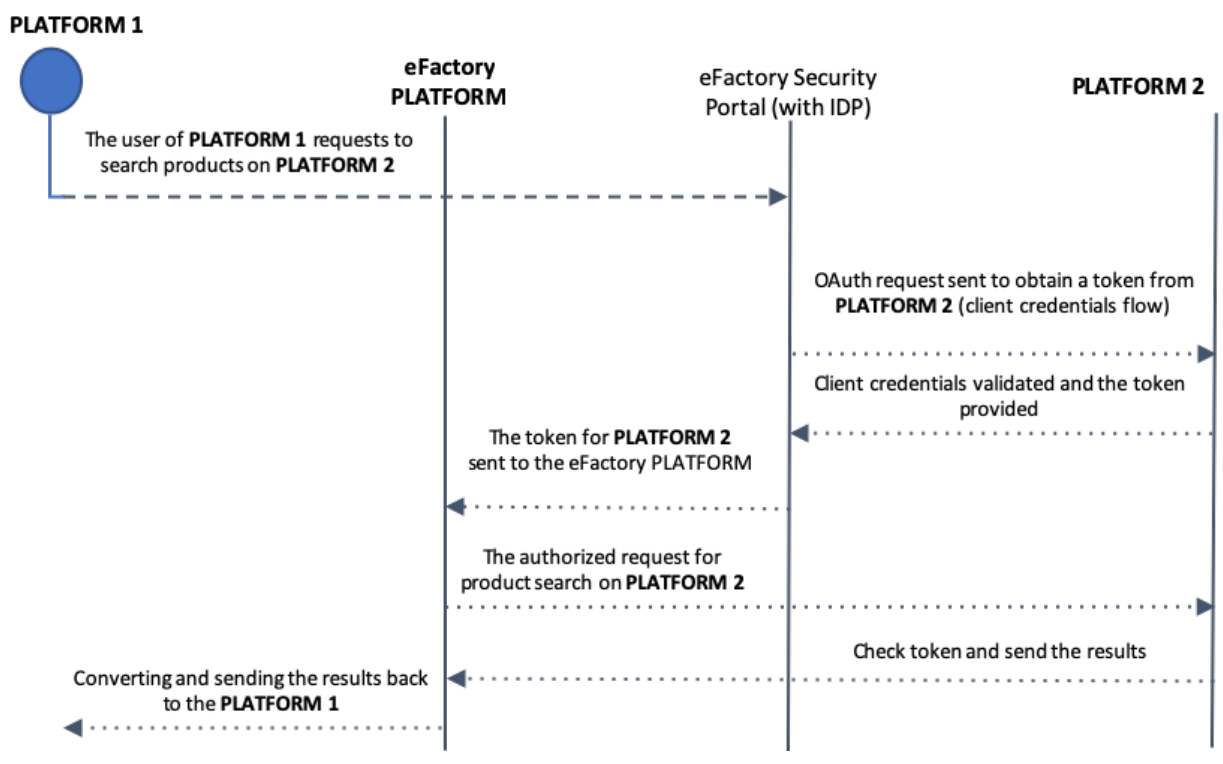

Figure 6: Integration level interoperability between several platforms in the eFactory ecosystem

\section{Conclusion Remarks and Next Steps}

In this paper, we follow the federated cloud identity broker model discussed in Section 2. Here, we describe two levels of interoperability to be achieved between the platforms, e.g. platform integration level interoperability and platform level interoperability. In addition, we describe two workflows for the platform level interoperability and their mechanisms to aggregate identities enabling the user to use the same identity information to access any service in the ecosystem. Our current research on designing and implementing the federated IDM in eFactory, enables the users to seamlessly login to other platforms in the eFactory ecosystem by complying to open standards.

Further work should be done to better understand the minimum level policies needed for the user in a specific platform to perform the same actions in the context of the eFactory federation. Further work should also be done to implement a policy engine in the integration middleware (Data Spine) to federate service calls, manage the access to the base platforms services, and provide a global IDM system for eFactory (through EFS), covering the stack from the network layer to high level application services.

\section{ACKNOWLEDGMENTS}

This research has been co-funded by the European Commission (European Union) within the H2020 FoF-ICT-2016 project NIMBLE (Collaborative Network for Industry, Manufacturing, Business and Logistics in Europe) No. 723810, and the H2020 DT-ICT-07-2018-2019 project eFactory (European Connected Factory Platform for Agile Manufacturing) No. 825075.

\section{REFERENCES}

[1] H2020 FoF-11-2016 eFactory (European Connected Factory Platform for Agile Manufacturing) project website: https://www.efactory-project.eu/

[2] B. Zwattendorfer, T. Zefferer and K. Stranacher, 2014. An Overview of Cloud Identity Management-Models. In Proceedings of the 10th International Conf. on Web Information Systems and Technologies (WEBIST), pp. 82-92.

[3] A. Jøsang and S. Pope, 2005. User centric identity management. AusCERT'05.

[4] E. Bertino, and K. Takahashi, 2011. Identity Management: Concepts, Technologies, and Systems. Artech House.

[5] Y. Cao, and L. Yang, 2010. A survey of Identity Management technology. In Proceedings of the IEEE ICITIS 2010, pp. 287-293. IEEE.

[6] C. Neuman, T. Yu, S. Hartman, and K. Raeburn, 2005. The Kerberos Network Authentication Service (V5). RFC 4120 (Proposed Standard).

[7] M. Dabrowski, and P. Pacyna, 2008. Overview of Identity Management. Technical report, chinacommunications.cn. 
Federated Identity Management and Interoperability for Heterogenous Cloud Platform Ecosystems, ARES'19, August 2629, 2019, Canterbury, United Kingdom

[8] H. Leitold, A. Hollosi, and R. Posch, 2002. Security architecture of the Austrian citizen card concept. In ACSAC 2002, pp. 391-400.

[9] J. Fromm, and P. Hoepner, 2011. The New German eID Card. In Fumy, W. and Paeschke, M. (Eds.), Handbook of eID Security - Concepts, Practical Experiences, Technologies, pp. 154-166. Publicis Publishing, Erlangen.

[10] J. Palfrey and U. Gasser, 2007. CASE STUDY: Digital Identity Interoperability and e-Innovation. Berkman Publication Series.

[11] C. Kaler and M. McIntosh, 2009. Web Services Federation Language (WSFederation) Version 1.2. OASIS Standard.

[12] SAML XML.org. Website: http://saml.xml.org/

[13] Shibboleth Consortium Website Online available from: https://www.shibboleth.net/products/identity-provider/ (accessed: May 2019)

[14] S.E. Haddouti and M.D.E.-C. El Kettan, 2015. Towards an Interoperable Identity Management Framework: A Comparative Study. International Journal of Computer Science Issues (IJCSI), Vol. 12, Issue 6. ISSN: 1694-0814. Online: https://arxiv.org/ftp/arxiv/papers/1902/1902.11184.pdf

[15] M. Ates, S. Ravet, A.M. Ahmat, and J. Fayolle, 2011. An Identity-Centric Internet: Identity in the Cloud, Identity as a Service and Other Delights. ARES 2011, pp. 555-560.

[16] Cloud Security Alliance, 2011. Security Guidance for Critical Areas of Focus in Cloud Computing V3.0. CSA
N. Selvanathan et al.

[17] H.Y. Huang, B. Wang, X.X. Liu, and J.M. Xu, 2010. Identity Federation Broker for Service Cloud. ICSS 2010, pp. 115-120.

[18] B. Zwattendorfer, K. Stranacher, and A. Tauber, 2013. Towards a Federated Identity as a Service Model. In Egovis 2013, pp. 43-57.

[19] D. Nunez, I. Agudo, and J. Lopez, 2012. Integrating OpenID with Proxy ReEncryption to enhance privacy in cloud-based identity services. In IEEE CloudCom 2012, pp. $241-248$.

[20] D. Nunez and I. Agudo, 2014. Blindidm: A privacy-preserving approach for identity management as a service. International Journal of Information Security Vol. 13, Issue 2, pp. 199-215. Online: https://link.springer.com/article/10.1007/s10207-014-0230-4

[21] M. Green and G. Ateniese, 2007. Identity-Based Proxy Re-encryption. In ACNS 2007, Vol. 4521 of LNCS, pp. 288-306. Springer.

[22] G. Ateniese, K. Fu, M. Green, and S. Hohenberger, 2006. Improved proxy reencryption schemes with applications to secure distributed storage. ACM Trans. Inf. Syst. Secur., 9(1):1-30.

[23] B. Zwattendorfer, 2014. Towards a Privacy-Preserving Federated Identity as a Service Model. PhD Thesis. The University of Graz, Austria.

[24] SOCIoTAL, 2014. D2.2 Framework Specification for Privacy and Access Control. Online: http://bit.ly/2B8HeB8 